\title{
Nation-wide analysis of the impact of Covid-19 pandemic on daily urology practice in Turkey
}

Ozan Bozkurt ${ }^{1}$, Volkan Sen ${ }^{2}$, Bora Irer ${ }^{3}$, Levent Sagnak ${ }^{3}$, Bulent Onal ${ }^{3}$, Yiloren Tanidir ${ }^{3}$, Emre Karabay ${ }^{4}$, Coskun Kaya ${ }^{5}$, Erman Ceyhan ${ }^{3}$, Aykut Baser ${ }^{3}$, Mesut Berkan Duran $^{3}$, Evren Suer ${ }^{6}$, Ilker Celen ${ }^{3}$, Ismail Selvi ${ }^{7}$, Oktay Ucer ${ }^{3}$, Sedat Karakoc ${ }^{3}$, Ege Sarikaya ${ }^{3}$, Ender Ozden ${ }^{3}$, Dogan Deger ${ }^{3}$, Sedat Egriboyun ${ }^{3}$, Sakir Ongun ${ }^{8}$, Ozgur Gurboga ${ }^{3}$, Mehmet Kazim Asutay ${ }^{3}$, Ilke Onur Kazaz ${ }^{9}$, Ismail Onder Yilmaz ${ }^{3}$, Erdem Kisa ${ }^{10}$, Engin Denizhan Demirkiran $^{3}$, Ozan Horsanali ${ }^{3}$, Ilker Akarken ${ }^{3}$, Onur Kizer ${ }^{3}$, Huseyin Eren ${ }^{11}$, Murat Uçar ${ }^{12}$, Oguz Ozden Cebeci ${ }^{13}$, Fuat Kizilay ${ }^{3}$, Kaan Comez ${ }^{3}$, Mehmet Mercimek ${ }^{14}$, Mehmet Serkan Ozkent $^{3}$, Volkan Izol ${ }^{15}$, Ahmet Gudeloglu ${ }^{3}$, Bilgin Ozturk ${ }^{3}$, Kaan Akbaba ${ }^{3}$, Salih Polat ${ }^{3}$, Adnan Gucuk ${ }^{3}$, Avni Ziyan ${ }^{3}$, Berin Selcuk ${ }^{3}$, Firat Akdeniz ${ }^{3}$, Hasan Turgut ${ }^{3}$, Kubilay Sabuncu $^{16}$, Onur Kaygisiz ${ }^{3}$, Veli Ersahin ${ }^{3}$, Halil Ibrahim Kahraman ${ }^{3}$, Muhammet Guzelsoy $^{3}$, and Omer Demir ${ }^{3}$

${ }^{1}$ Dokuz Eylül University

${ }^{2}$ Manisa State Hospital

${ }^{3}$ Affiliation not available

${ }^{4}$ Istsanbul Haydarpasa Numune Training and Research Hospital

${ }^{5}$ Eskisehir City Hospital, Department of Urology

${ }^{6}$ Ankara University Faculty of Medicine

${ }^{7}$ Oncology Training and Research Hospital

${ }^{8}$ Balikesir University

${ }^{9}$ Karadeniz Technical University

${ }^{10}$ Izmir Tepecik Training and Research Hospital

${ }^{11}$ Recep Tayyip Erdoğan University

${ }^{12}$ Faculty of Medicine, Alanya Alaaddin Keykubat University

${ }^{13}$ Saglik Bilimleri Universitesi

${ }^{14}$ Liv Hospital Samsun

${ }^{15}$ Cukurova University Faculty of Medicine

${ }^{16}$ Dr Lutfi Kirdar Training

September 11, 2020

\begin{abstract}
Objective: To present a nation-wide analysis of the workload of urology departments in Turkey week-by-week during Covid-19 pandemic. Methodology: The centers participating in the study were divided into three groups as tertiary referral centers, state hospitals and private practice hospitals. The number of outpatients, inpatients, daily interventions and urological surgeries were recorded prospectively between 9-March-2020 and 31-May-2020. All these variables were recorded for the same time interval of 2019 as well. The weekly change of the workload of urology during pandemic period was evaluated; also the workload of urology and the distributions of certain urological surgeries were compared between the pandemic period and the
\end{abstract}


same time interval of the year 2019. Results: A total of 51 centers participated in the study. The number of outpatients, inpatients, urological surgeries and daily interventions were found to be dramatically decreased by the third week of pandemics in state hospitals and tertiary referral centers; however the daily urological practice were similar in private practice hospitals throughout the pandemic period. When the workload of urology in pandemic period and the same time interval of the year 2019 were compared; a huge decrease was observed in all variables during pandemic period. However, temporary measures like ureteral stenting, nephrostomy placement and percutaneous cystostomy have been found to increase during Covid-19 pandemic compared to normal life. Conclusions: Covid-19 pandemic significantly effected the routine daily urological practice likewise other subspecialties and priority was given to emergent and non-deferrable surgeries by urologists in concordance with published clinical guidelines.

\section{Introduction}

Coronaviruses $(\mathrm{CoV})$ are a large family of RNA viruses which can lead to various infections from self-limiting mild upper respiratory infections to serious life-threatening infections like Middle East Respiratory Syndrome (MERS) and Severe Acute Respiratory Syndrome (SARS). ${ }^{1-3}$ At December 2019, cases of pneumonia with unknown etiology were reported in Wuhan, Hubei province of China. ${ }^{4}$ At the beginning of the January 2020; the 2019 novel coronavirus (2019-nCoV) was isolated and World Health Organization (WHO) named this virus as Severe Acute Respiratory Syndrome Coronavirus 2 (SARS-CoV-2) and the disease resulting with pneumonia as Coronavirus Disease 2019 (Covid-19). ${ }^{2}$ Covid-19 spread rapidly from country to country and as a result WHO declared Covid-19 as a pandemic on the March $11^{\text {th }} 2020 .^{5}$ Worldwide total cases and the number of deaths reached 14.348 .858 and 603.691, respectively. ${ }^{6}$ The first case in Turkey was reported at 11 March 2020 and the peak of daily cases and deaths were seen during the $4^{\text {th }}$ week of the pandemic curve in our country. ${ }^{6}$

The workload of hospitals has increased considerably during the pandemic process and many healthcare measures were taken by governments and hospital systems. Most of the hospitals turned to pandemic or quarantine hospitals and had to serve only Covid-19 patients. Some detailed recommendations were published for the triage of urological surgeries during the Covid-19 pandemic. ${ }^{7-10}$ Like other surgical subspecialties; cancellation of elective surgeries and utilization of solely emergent surgeries and non-deferrable oncologic surgeries that delay may cause negative impact on survival have been performed in urology clinics according the triage recommendations. ${ }^{11}$ Also, urological outpatient clinics have been adapted to new social distancing rules in Covid-19 pandemic; the number of appointments was limited and only emergent patients could be treated in most of the urology clinics in worldwide as in our country. $8,12,13$

In this report, we aimed to present a nation-wide analysis of the workload of urology departments in Turkey week-by-week during Covid-19 pandemic and to compare the outcomes with the same time interval of the year 2019.

\section{Methods}

The study was approved by the local ethics committee. An announcement of the study was sent to all Urology clinics across Turkey via e-mail and social media. All of the centers that agreed to participate in the study were included in the study. The number of outpatient and hospitalized patients, emergency service consultations, urological surgeries including transurethral resection of bladder tumor (TUR-BT), transurethral resection of prostate (TURP), endoscopic urethrotomy, ureterorenoscopy (URS), percutaneous nephrolithotomy (PNL), ureteral J stent insertion, radical nephrectomy, radical nephroureterectomy, radical prostatectomy (RP), radical cystectomy $(\mathrm{RC})$, radical orchiectomy, surgery for Fournier's gangrene, acute scrotum, trauma (kidney, bladder and testicle) and penile fracture; daily interventions (prostate biopsy, shock wave lithotripsy, percutaneous nephrostomy and cystostomy, intravesical treatments), and the number of pandemic outpatient clinics were recorded week-by-week between the week that the first Covid-19 patient was reported in our country, 9-15 March 2020, and the week 25-31 May 2020 prospectively; as lock-down measures were gone and the 'new normal-life' began in our country by $1^{\text {st }}$ June 2020 . All of these variables were also recorded week-by-week at the same time interval of the year 2019. Other surgical and diagnostic approaches were not analyzed as guidelines regarding Covid-19 pandemic suggested the postponement of nearly all 
surgeries for female urology, andrology and some other elective surgical operations. ${ }^{8,11-13}$ The centers were divided into three groups as tertiary referral centers, state hospitals and private practice hospitals. The weekly change of the workload of urology throughout the pandemic period was evaluated; also the workload of urology and the distributions of certain urological surgeries were compared between the pandemic period and the same time interval of the year 2019.

\section{Statistical analysis}

All statistical analyses were performed with the Statistical Package for the Social Sciences (SPSS, Inc., Chicago IL), version 22, software for Windows. Shapiro-Wilk test was used to determine whether the data was normally distributed, since p values were found to be greater than 0.05 , it was decided that the data were normally distributed. After the descriptive statistics were made, the data for the 2019 period and pandemic period were compared with the Paired-Samples $T$ test. The results were given as mean \pm standard deviation and $\mathrm{n}(\%) . \mathrm{P}<0.05$ was considered statistically significant.

\section{Results}

A total of 51 centers from all geographical areas participated in the study. Of these centers; $30(58.8 \%)$ were tertiary referral centers, $15(29.4 \%)$ were state hospitals and $6(11.8 \%)$ were private practice hospitals. The number of outpatients, inpatients, urological surgeries and daily interventions were found to be dramatically decreased by the third week of pandemics in state hospitals and tertiary referral centers; however the daily practice were similar in private practice hospitals throughout the pandemic period. Urooncological surgeries were decreased week by week in tertiary referral centers; a huge decrease was observed for the stone surgeries at the third week of the pandemics in tertiary referral centers and state hospitals; however the number of emergent/trauma surgeries was relatively similar in both centers during the pandemics. The weekly analysis of workload of urology and urological surgeries by categories were given in Figure 1 and Figure 2 .

When the workload of urology in pandemic period and the same time interval of the year 2019 were compared; a huge decrease was observed in all variables during pandemic period (Table 1). In a detailed analysis of the three groups of centers; a significant decrease was detected in outpatients, inpatients, daily interventions and urological surgeries in tertiary referral centers and state hospitals; however the decrease was not statistically significant in private practice hospital in terms of inpatients and daily interventions (Figure 3). The numbers of urooncological, stone, $\mathrm{BPH}$ and emergent/trauma surgeries were significantly decreased in tertiary referral centers and state hospitals in pandemic period compared with the same time interval of the year 2019; but the numbers of urooncological and stone surgeries were similar in private practice hospitals (Figure 4). The distributions of the urooncological and stone surgeries in pandemic period and the same time interval of 2019 were given in Figure 5. While the numbers of urooncological surgeries decreased dramatically in pandemic period; the distribution of urooncological surgeries was similar between the pandemic period and the year 2019 (Figure 5). The highest decrease in urooncological surgeries was detected in nephroureterectomy (338 to $25 ;-92.7 \%$ ); followed by radical-partial nephrectomy operations (606 to $121 ;-80.1 \%$ ), orchiectomy (288 to 58 ; $-79.9 \%$ ), TURBT (3329 to $742 ;-77.8 \%$ ), radical prostatectomy (440 to $103 ;-76.6 \%$ ), and radical cystectomy (223 to $61 ;-62.7 \%$ ). The number of percutaneous nephrolithotomy and ureterorenoscopy dropped with the rates of $81.1 \%$ ( 822 to 156 ) and $73.0 \%$ (3728 to 1010) in pandemic period. While the total count of ureteral $\mathrm{J}$ stent insertion decreased as $70.4 \%$ (3279 to 972 procedures); the rate of this procedure has increased with a rate of $4 \%(42 \%$ to $46 \%)$ in pandemic period (Figure 5 ).

Although the total numbers of temporary measures like nephrostomy placement (539 to $223 ;-58.7 \%$ ) and percutaneous cystostomy ( 661 to $253 ;-61.8 \%$ ) for certain instances decreased; the rate of these procedures for emergent-trauma surgeries has been found to increase during pandemic period ( $15 \%$ to $22.7 \%$ and $18.4 \%$ to $25.7 \%$; respectively).

Urologists took active role in fighting against Covid-19 with all of the centers participating in this study; $54.4 \pm 100.7,41.0 \pm 34.6,10.0 \pm 24.5$ pandemic outpatient clinics were performed by urologist in tertiary referral centers, state hospitals and private practice hospitals, respectively. 


\section{Discussion:}

Mass casualty events including natural disasters (earthquakes, floods, and landslides), biological, chemical, nuclear and radiological disasters lead to increase the requirement for healthcare. Virus pandemics are a kind of biological disasters and the best known virus pandemics were Spanish Flu pandemics which killed over 20 million people from 1918 to 1919, SARS-CoV pandemics that affected approximately 8000 people with a mortality rate $10 \%$ and MERS-CoV pandemics which affected over 800 people with a mortality rate of $35 \%{ }^{8,14}$ Therefore, it is essential to enhance the capabilities of healthcare institutions, for mitigation of disasters' effects. Governments and healthcare institutions must prepare their virus pandemic plans, to be able to intervene in time for pandemics. A sample of pandemic influenza planning of the state of Connecticut was reported by Duley MG at 2005. ${ }^{15}$ Some of the recommendations of this report to assure health care facility were; suspending all of the elective outpatient and inpatient surgeries and procedures, developing strategies to increase bed availability for influenza patients and implementing triage to reduce non-influenza admissions. ${ }^{16}$ Covid-19 first appeared in China and spread rapidly between the countries, and was declared as pandemics at 11-March-2020 by WHO. Similar with the recommendations of pandemics plan of the State Connecticut, many countries took measures stage by stage for the Covid-19 pandemic. Like in the other specialties, several guidelines and measures were published for urology practice during Covid-19 pandemic. ${ }^{7-11,16}$ Wallis et al and Stensland et al published review and editorial articles about the triage and the management of genitourinary cancers and the risks of delaying in treatment. ${ }^{7,16}$ Their recommendations for the treatment of urological cancers during Covid-19 era were as: transurethral resection of bladder tumor (TURBT) can be performed in high-grade non-muscle-invasive bladder cancer (NMIBC) however cystoscopic surveillance and (TURBT) for recurrence in patients with known low-grade NMIBC can be deferred; the initial treatment of high-grade NMIBC should be the induction Bacillus Calmette-Guérin (BCG) and a single course of maintenance therapy $(6+3)$; over than 12 weeks delay in radical cystectomy $(\mathrm{RC})$ was found associated with decreased overall and progression-free survival ${ }^{17}$ so RC should be prioritized; active surveillance should be the first option for low-risk prostate cancer; it was reported that delaying 3 to 6 months for the treatment of intermediate and high-risk prostate cancer patients was not associated with adverse biochemical recurrence, pathological and survival outcomes. ${ }^{18}$ The radical prostatectomy and definitive therapies can be deferred; small renal masses can be safely observed with active surveillance, and the treatment of localized kidney cancers (cT1b and cT2 tumors) can be delayed to 3-6 months without adverse affects in outcomes; however radical nephrectomy should be performed in priority in locally advanced kidney cancers (cT3+); the risk of a delay in the treatment of upper tract urothelial cancer (UTUC) is depended on the stage and grade of cancer, especially in high-grade UTUC a delay up-to 3 months was found associated with disease progression, ${ }^{19}$ so keep in mind nephroureterectomy in these patients; avoid from delaying radical orchiectomy in testicular cancer patients; avoid from a delay in penile cancer treatments including surgeries. ${ }^{16}$ Harmoniously with these recommendations, Pinar et al reported a decrease of $31 \%$ in the surgeries of genitor-urinary cancers in comparison with the same time interval of 2019 (12-27 March) and they performed un-deferrable oncological surgeries in 8 academic urological departments of Paris, France. ${ }^{20}$ Tinay et al evaluated the early impact of Covid-19 on surgical urologic oncology practice in several tertiary institutions of Turkey, and they compared the outcomes of early pandemic period (March-11 to April-11) with the same time interval of $2019 .{ }^{11}$ They detected a decrease (from 200 to 90 cases) in the numbers of urothelial carcinoma, kidney cancer and prostate cancer surgeries, and they pointed that the centralization of oncological surgeries is required during the disasters like this pandemic. A significant decrease was also detected in all of the oncologic surgical procedures during pandemic period in our study (-78.8\%). As stated above, most of the urooncological surgeries are usually performed in tertiary referral centers in our country; so the highest decrease has been observed in these centers whereas no significant change was observed in private practice hospitals in terms of urooncological surgery. The lowest decrease in urooncological surgeries was detected in radical cystectomy operations in the present study as most of the above-mentioned reports and guidelines offer prioritization of radical cystectomy. ${ }^{8,16}$ We detected a sharp decrease within $3^{\text {rd }}$ and $4^{\text {th }}$ week of the pandemic in our country for all urological as well as urooncological cases as government and healthcare authorities suggested lock-down measures for the spread of SARS-CoV-2; and those low case load continued until the end of our study period which reflects the end of lock-down measures in our country. An interesting finding of this 
study was that the ratio of almost all urooncological surgeries was similar between the pandemic period and routine daily practice despite a significant decrease in total numbers. We did not deeply analyze the surgical indications, but surgery for higher risk cases for all cancer types might have been prioritized in most involved centers. TUR-BT was the mostly utilized urooncological surgical procedure for both the pandemic period and normal life conditions in our country in concordance with previous reports. ${ }^{11}$

Cancellation of elective surgeries for urolithiasis, benign prostatic hyperplasia and urethral strictures were recommended in pandemic period. ${ }^{7}$ If there is an obstruction in upper urinary tract, the ureteral stenting or nephrostomy tube placement are recommended instead of definitive treatments. ${ }^{7,21,22}$ The EULIS Collaborative Research Working Group published the results of a survey related with routine practice of endourologists in stone diseases during the Covid-19 pandemic. ${ }^{23}$ They pointed that the majority of the participants $(89.4 \%)$ have used to perform temporary interventions like JJ placement or percutaneous nephrostomy, rather than the stone removal operations. ${ }^{23} \mathrm{Gul}$ et al reported that complicated ureteral stone diseases have increased in pandemic period; consequently the rate of nephrostomy placement has also increased. ${ }^{24}$ In accordance with the recommendations; the numbers of elective surgeries (URS and PNL) for urolithiasis, benign prostatic diseases and endoscopic urethrotomies have decreased and the rates of temporary measures such as ureteral stenting, nephrostomy placement and percutaneous cystostomy as emergent interventions have increased during pandemic period in our study. This reflects to the adaptation of urologic surgeons in our country to the published recommendations. ${ }^{7,21,23}$

The healthcare institutions have to prepare strategies to increase bed resources and availability for Covid19 patients. The main measures for this process include; performing the triage in outpatient clinics to decrease other types of admissions and decreasing the number and length of hospital stay. ${ }^{15}$ The healthcare institutions decreased their outpatient and inpatient clinics according to recommendations of the Ministry of Health of Turkey in our country. Due to the measures; the number of patients admitted to the outpatient clinics decreased with a rate of $73.7 \%$ and the number of the patients which were treated inpatient decreased with a rate of $71.3 \%$ during pandemic period in comparison with the same time interval of 2019 in urology departments participated in this study. Those measures should undoubtedly be taken by managers and chief executive officers. The difference adaptation of tertiary referral centers, state hospitals and private practice hospitals with regard to these measures may come from different management options. Pandemic patients were primarily treated by state hospitals and tertiary referral centers in our country, so the elective cases were cancelled by these hospital types whereas most private practice hospitals did not treat Covid-19 patients.

Our findings demonstrated that workload for urological diseases dramatically decreased during Covid-19 pandemic. However, the workload of hospitals dramatically increased during the Covid-19 pandemic and most of the hospitals had to turn to pandemic or quarantine hospitals and serve only Covid-19 patients. A total of 2307 pandemic outpatient clinics by 8-hour shifts were done by urologists in our study. So, we detected that urologists also took active role in the front-line management of Covid-19 patients in our country.

Participation of mainly tertiary referral centers in comparison with state and private practice hospitals constitute one of the limitations of the present study. Most state hospitals also turned to pandemic hospitals and did not serve for routine practice. Participation of more state hospitals would better reflect daily practice. However, 2019 results demonstrated that most of the Urology workload was met by tertiary referral centers in our country.

Conclusions: Covid-19 pandemic led to a serious challenge to healthcare systems. Like the worldwide results; the number of outpatients, inpatients and daily interventions have decreased, elective surgeries mostly deferred and a priority has given to emergent and high-grade malignancy surgeries in our country. We believe that the results of the present study will help in organization of human resources and triage of urology clinics for further possible mass casualty events.

Conflict of interest: none declared.

Acknowledgements: none declared. 


\section{References}

1. Cheng VC, Lau SK, Woo PC, Yuen KY. Severe acute respiratory syndrome coronavirus as an agent of emerging and reemerging infection. Clin Microbiol Rev. 2007;20:660-694.

2. He F, Deng Y, Li W. Coronavirus disease 2019: What we know? [published online ahead of print, 2020 Mar 14]. J Med Virol. 2020;10.1002/jmv.25766.

3. Zumla A, Chan JF, Azhar EI, Hui DS, Yuen KY. Coronaviruses - drug discovery and therapeutic options. Nat Rev Drug Discov. 2016;15:327-347.

4. Bogoch II, Watts A, Thomas-Bachli A, Huber C, Kraemer MUG, Khan K. Pneumonia of unknown aetiology in Wuhan, China: potential for international spread via commercial air travel. J Travel Med. 2020;27:taaa008.

5. World Health Organization. Coronavirus disease 2019 (COVID-19) Situation Report -51 [Internet]. 11 March. 2020 [cited 2020 May 25]. Available from:https://www.who.int/docs/defaultsource/coronaviruse/situation-reports/20200311-sitrep-51-covid-19.pdf?sfvrsn=1ba62e57_10.

6. World Health Organization. Coronavirus disease 2019 (COVID-19) Situation Report -182 [Internet]. 20 July 2020 [cited 2020 July 21]. Available from: https://www.who.int/docs/defaultsource/coronaviruse/situation-reports/20200720-covid-19-sitrep-182.pdf?sfvrsn=60aabc5c_2

7. Stensland KD, Morgan TM, Moinzadeh A, et al. Considerations in the Triage of Urologic Surgeries During the COVID-19 Pandemic [published online ahead of print, 2020 Apr 9]. Eur Urol. 2020;S0302-2838(20)302025. doi:10.1016/j.eururo.2020.03.027.

8. Puliatti S, Eissa A, Eissa R, et al. COVID-19 and urology: a comprehensive review of the literature [published online ahead of print, 2020 Apr 6]. BJU Int. 2020;10.1111/bju.15071. doi:10.1111/bju.15071.

9. Margel D, Ber Y. Changes in Urology After the First Wave of the COVID-19 Pandemic [published online ahead of print, 2020 May 13]. Eur Urol Focus. 2020;10.1016/j.euf.2020.05.001. doi:10.1016/j.euf.2020.05.001

10. Ribal MJ, Cornford P, Briganti A, et al. European Association of Urology Guidelines Office Rapid Reaction Group: An Organisation-wide Collaborative Effort to Adapt the European Association of Urology Guidelines Recommendations to the Coronavirus Disease 2019 Era [published online ahead of print, 2020 Apr 27]. Eur Urol. 2020;S0302-2838(20)30324-9. doi:10.1016/j.eururo.2020.04.056

11. Tinay I, Ozden E, Suer E, et al. The Early Impact of COVID-19 Pandemic on Surgical Urologic Oncology Practice in Turkey: Multi-Institutional Experience from Different Geographic Areas [published online ahead of print, 2020 May 20]. Urology. 2020;S0090-4295(20)30567-7. doi:10.1016/j.urology.2020.05.016.

12. Margel D, Ber Y. Changes in Urology After the First Wave of the COVID-19 Pandemic [published online ahead of print, 2020 May 13]. Eur Urol Focus. 2020;10.1016/j.euf.2020.05.001. doi:10.1016/j.euf.2020.05.001.

13. Tan YQ, Wu QH, Chiong E. Preserving Operational Capability While Building Capacity During the COVID-19 Pandemic: A Tertiary Urology Centre's Experience [published online ahead of print, 2020 Apr 29]. Urology. 2020;S0090-4295(20)30500-8. doi:10.1016/j.urology.2020.04.079.

14. Taubenberger J.K., Morens D.M. Influenza: the Mother of All Pandemics. In. Emerg Infect Dis. 1918;2006:15-22.

15. Duley MG. The next pandemic: anticipating an overwhelmed health care system. Yale J Biol Med. 2005;78:355-362.

16. Wallis CJD, Novara G, Marandino L, et al. Risks from Deferring Treatment for Genitourinary Cancers: A Collaborative Review to Aid Triage and Management During the COVID-19 Pandemic [published online ahead of print, 2020 May 3]. Eur Urol. 2020;S0302-2838(20)30331-6. doi:10.1016/j.eururo.2020.04.063. 
17. Boeri L, Soligo M, Frank I, et al. Delaying radical cystectomy after neoadjuvant chemotherapy for muscle-invasive bladder cancer is associated with adverse survival outcomes. Eur Urol Oncol 2019;2:390-6.

18. Fossati N, Rossi MS, Cucchiara V, et al. Evaluating the effect of time from prostate cancer diagnosis to radical prostatectomy on cancer control: can surgery be postponed safely? Urol Oncol 2017;35:e9-150 e15.

19. Waldert M, Karakiewicz PI, Raman JD, et al. A delay in radical neph- roureterectomycan lead to upstaging. BJU Int 2010;105:812-7. http:// dx.doi.org/10.1111/j.1464-410X.2009.08821.x.

20. Pinar U, Anract J, Duquesne I, et al. Impact de la pandemie de COVID-19 sur l'activite chirurgicale au sein des services d'urologie de l'Assistance Publique - Hopitaux de Paris [Impact of the COVID-19 pandemic on surgical activity within academic urological departments in Paris] [published online ahead of print, 2020 May 11]. Prog Urol. 2020;S1166-7087(20)30148-2. doi:10.1016/j.purol.2020.05.001

21. Socarras MER, Esperto F, Bapstistussi MD, et al. Endourology (Lithiasis). Management, surgical considerations and follow-up of patients in the COVID-19 era. Int Braz J Urol. 2020;46(suppl.1):39-49. doi:10.1590/S1677-5538.IBJU.2020.S105.

22. Proietti S, Gaboardi F, Giusti G. Endourological Stone Management in the Era of the COVID-19. Eur Urol. 2020;78(2):131-133. doi:10.1016/j.eururo.2020.03.042.

23. Tefik T, Guven S, Villa L, et al. Urolithiasis Practice Patterns Following the COVID-19 Pandemic: Overview from the EULIS Collaborative Research Working Group [published online ahead of print, 2020 Apr 27]. Eur Urol. 2020;S0302-2838(20)30325-0. doi:10.1016/j.eururo.2020.04.057.

24. Gul M, Kaynar M, Yildiz M, et al. The Increased Risk of Complicated Ureteral Stones in the Era of COVID-19 Pandemic [published online ahead of print, 2020 Jul 29]. J Endourol. 2020;10.1089/end.2020.0658. doi:10.1089/end.2020.0658

\section{Figure legends}

Figure 1. Week-by-week analysis of the workload of urology during Covid-19 pandemic. Mean number values were provided for tertiary referral centers, state hospitals and private practice hospitals. a. Outpatient clinics; b. Inpatients during the pandemic; c. Emergency service consultations; d. Total number of Urological Surgeries; e. All daily interventions including intravesical administrations, prostate biopsies, dilatations etc.; f. Weekly mean working hour of Urologists for pandemic outpatient clinics.

Figure 2. Weekly analysis of urological surgeries by categories a. Average case numbers for Urooncological surgery dropped to $1,8+-1,4$ cases from $8.5+-7.8$ cases by the $4^{\text {th }}$ week compared to the $1^{\text {st }}$ week; $b$. Mean number of stone surgeries declining by $3^{\text {rd }}$ week and a plateau during the pandemic; c. Surgery for benign prostate hyperplasia dropped to zero by $6^{\text {th }}$ week for tertiary referral centers and state hospitals ; $d$. Emergent-trauma surgery also declined during this pandemic.

Figure 3. a. Average outpatient numbers significantly declined during the Covid-19 pandemic compared with $2019(8330.7+-4727.3$ to $2306.0+-1330$ for tertiary referral centers $(p<0.001) ; 6580.9+-5408.3$ to $1424.9+-874.5$ for state hospitals $(p=0.001)$ and $900.3+-337.2$ to $427.7+-303.0$ for private practice hospitals $(p=0.012))$ b. Hospitalized patients in Urology clinics significantly decreased for tertiary referral centers and state hospitals; $508.5+-402.8$ to $148.3+-95$. $(p<0.001)$ and $171.6+-149.7$ to $34.5+-26.5(p=0.001)$, respectively. These numbers also decreased for private practice hospitals without statistical significance and $98.3+-28.6$ to $55.0+-43.7$ ( $p=0.052$ ) c. Emergency consultations were similar between the pandemic and 2019 period for state hospitals and private practice hospitals $(74.3+-61.3$ to $68.0+-67.1,(p=0.721)$ and $15.3+-8.9$ to $9.0+-13.1,(p=0.128))$ whereas significantly decreased in tertiary referral centers $(225.8+-186.8$ to $161.4+-158.4, p=0.018)$ d. All urologic surgical activities significantly declined compared to previous year during the pandemic period for all centers. (591.2+-815.5 to 117.2+-64.4 for tertiary referral centers $(p=0.003)$; $173.2+-159.0$ to $32.2+-27.9$ for state $(p=0.001)$ and $88.3+-27.9$ to $48.8+-35.2$ for private practice hospitals $(p=0.043))$ e. Daily interventions were significantly reduced in tertiary referral centers and 
state hospitals, but not for private practice hospitals $(533.6+-956.2$ to $108.9+-210.4, p=0.005 ; 159.1+-140.1$ to $41.9+-38.4, p=0.007$; and $30.7+-13,0$ to $21.8+-13.8, p=0.292$ ).

Figure 4. Comparison of certain urological surgeries between the pandemic period and the same time interval of 2019. a. Considering all urooncological surgical procedures, there was a decline compared to previous year where the difference did not reach statistical significance for tertiary referral centers and private practice hospitals. (165.2+-432.2 to $32.4+-30.0, p=0.085 ; 12.2+-13.2$ to $3.7+-2.9, p=0.022$ and $14.3+-11.1$ to $12.8+-11.3, p=0.680$ for tertiary referral centers, state hospitals and private practice hospitals, respectively.) b. Surgery for stone disease was significantly reduced in tertiary referral centers $(210.1+-181.5$ to $57.5+-42.4, p<0.001)$ and state hospitals $(93.3+-122.2$ to $19.5+-27.0, p=0.011)$. Case-load was similar for private practice hospitals $(21.2+-2.0$ to $20.3+-13.2, p=0.880)$ regarding stone surgery. c. BPH surgery decreased significantly in all hospitals. (105.7+-210.1 to $26.6+-21.2, p=0.038 ; 25.5+-28.6$ to $10.7+-10.3$, $p=0.015 ; 6.3+-3.0$ to $3.3+-1.7, p=0.023$; for tertiary referral centers, state hospitals and private practice hospitals, respectively.) d. Emergent surgical procedures also declined in all centers compared to previous year $(46.2+-46.3$ to $7.1+-4.7, p<0.001 ; 15.0+-15.9$ to $1.5+-1.9, p=0.002 ; 8.3+-3.4$ to $3.2+-3.9, p=0.011$; for tertiary referral centers, state hospitals and private practice hospitals, respectively.)

Figure 5. The distribution of the urooncological surgeries and stone surgeries in 2019 and during the pandemic period

a. Urooncological surgery (2019) b. Urooncological surgery (pandemic period): The highest decrease in urooncological surgeries was detected in nephroureterectomy (338 to 25; -92.7\%); followed by radical-partial nephrectomy operations (606 to $121 ;-80.1 \%$ ), orchiectomy (288 to $58 ;-79.9 \%$ ), TURBT (3329 to 742 ; $-77.8 \%$ ), radical prostatectomy (440 to $103 ;-76.6 \%$ ), and radical cystectomy (223 to $61 ;-62.7 \%$ ).

c. Stone surgery (2019) d. Stone surgery (pandemic period): While the number of ureteral J stent insertion $70.4 \%$ (3279 to 972 ) decreased; the percentage of it in stone surgeries has increased with a rate of $4 \%$ ( $42 \%$ to $46 \%$ ) in pandemic period.

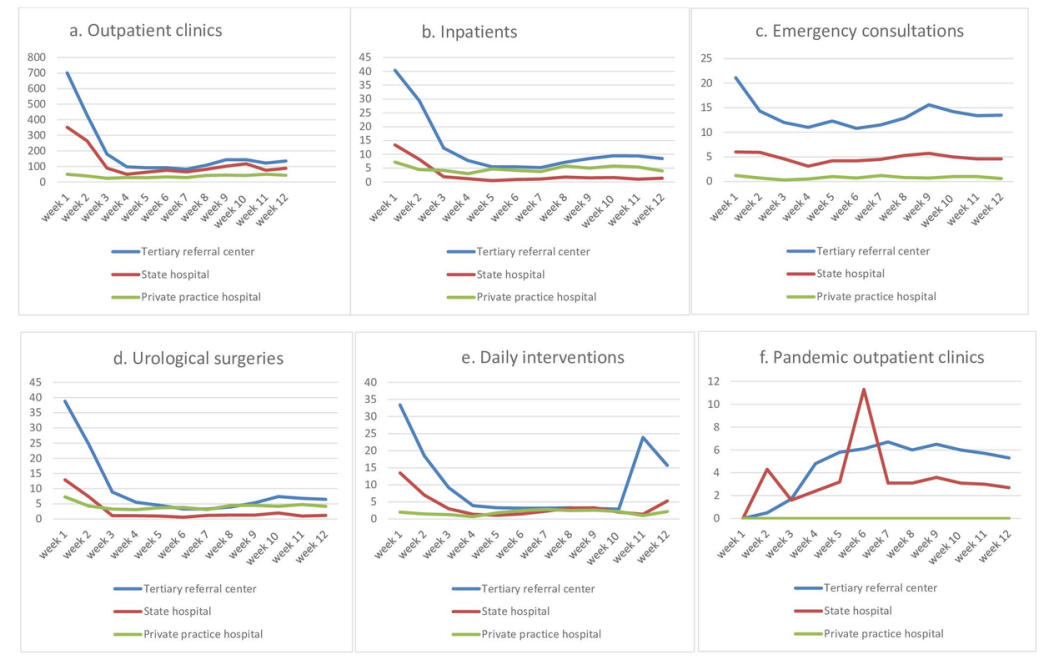



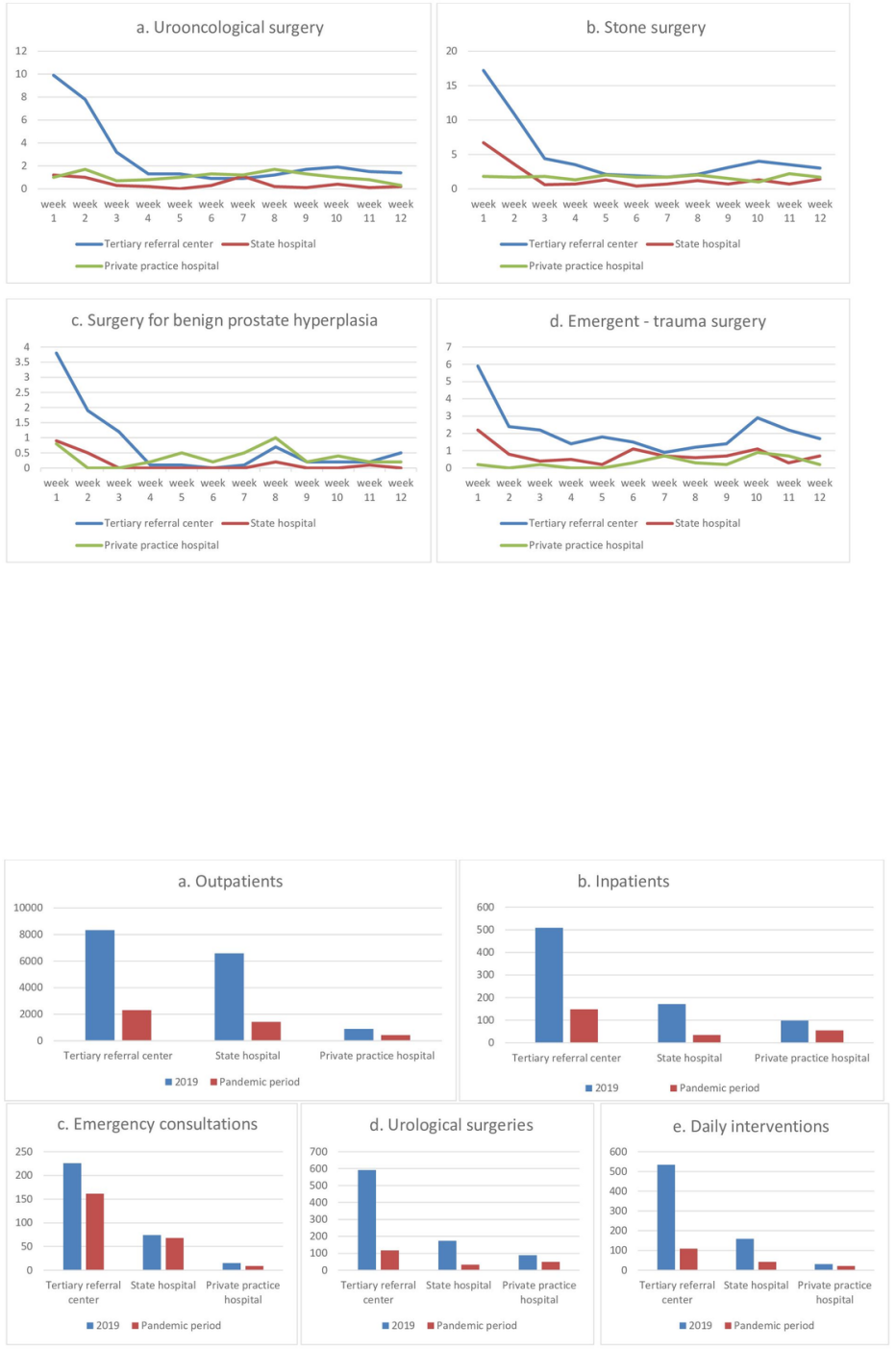

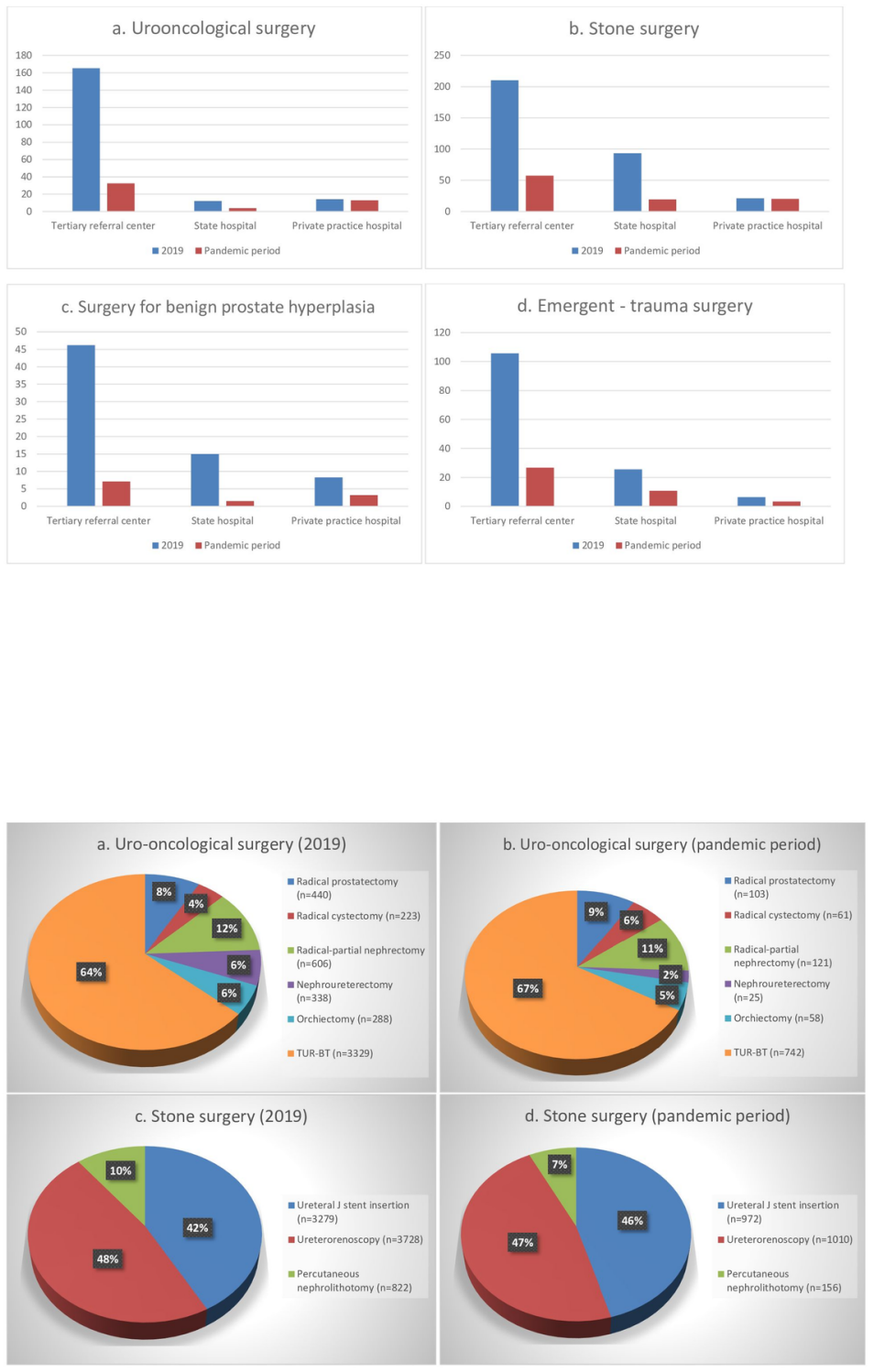

\section{Hosted file}

Table 1..docx available at https://authorea.com/users/353501/articles/480117-nation-wideanalysis-of-the-impact-of-covid-19-pandemic-on-daily-urology-practice-in-turkey 